Accepted in Research Notes of the AAS

\title{
THE MOIST GREENHOUSE IS SENSITIVE TO STRATOSPHERIC TEMPERATURE
}

\author{
Ramses M. Ramirez ${ }^{\mathrm{i}}$ \\ ${ }^{i}$ Earth-Life Science Institute, Tokyo, Japan \\ email: rramirez@elsi.jp
}

\section{INTRODUCTION}

Recent 3-D model simulations (Fujii et al. 2017; Kopparapu et al. 2017) (hereafter, F2017 and K2017) suggest that tidally-locked M-star planets at the inner edge of the habitable zone (HZ) undergo a moist greenhouse at significantly lower mean surface temperatures ( 280 $300 \mathrm{~K}$ ) than the $\sim 340 \mathrm{~K}$ predicted in 1-D models (e.g. Kasting et al., 1993). This moist greenhouse occurs once stratospheric $\mathrm{H}_{2} \mathrm{O}$ concentrations exceed $\sim 3 \times 10^{-3}$, when the cold trap is wet enough for atmospheric $\mathrm{H}_{2} \mathrm{O}$ to efficiently dissociate so that an amount of water equal to that in Earth's oceans can escape to space on $\sim 4.5 \mathrm{Gyr}$ timescales.

F2017 attribute these differences to strong near-infrared absorption (NIR) that triggers upward motions that increase upper atmospheric $\mathrm{H}_{2} \mathrm{O}$ concentrations more rapidly than predicted by 1-D models. F2017 also (correctly) argue that dynamical effects are very important to accurately characterize climates, which is something that 1-D models do not include explicitly (although this is often parameterized implicitly). Although true, this is not the main reason for the apparent discrepancy between 1-D and 3-D model results for calculated moist greenhouse thresholds on M-star planets.

It is known that the moist greenhouse mean surface temperature threshold is very sensitive to the stratospheric temperature (e.g. Kasting et al., 1993). In 1-D calculations (in lieu of knowing anything about the exoplanetary atmosphere), a 1-bar fully-saturated $\mathrm{N}_{2}$ atmosphere on a planet with an Earth-like surface water inventory and (most importantly) a constant stratospheric temperature of $200 \mathrm{~K}$ (above the cold trap) is traditionally assumed. However, the 3-D simulations in both F2017 and K2017 exhibit upper atmospheric and stratospheric temperatures well above $200 \mathrm{~K}$ as the moist greenhouse threshold is approached on their M-star planets. This note is a reminder of the importance of stratospheric temperatures for this particular calculation. 


\section{METHODS}

Here, I use my single-column climate model (e.g. Ramirez and Kaltenegger, 2017) to construct idealized cases that illustrate how the moist greenhouse surface temperature threshold changes as stratospheric temperature is increased. Atmospheres are fully-saturated and stratospheric temperature is assumed constant.

\section{RESULTS}

At a stratospheric temperature $\left(\mathrm{T}_{\text {strat }}\right)$ of $200 \mathrm{~K}$, the moist greenhouse is triggered at a mean surface temperature of $\sim 340 \mathrm{~K}$ (as expected) (Figure 1). However, it commences at significantly lower mean surface temperatures as $\mathrm{T}_{\text {strat }}$ is increased. At a $\mathrm{T}_{\text {strat }}$ of $(220,243,260) \mathrm{K}$, the moist greenhouse is triggered at surface temperatures of $\sim(330,309,280) \mathrm{K}$ (Figure). The latter two cases are consistent with the bulk trends of K2017 and F2017.
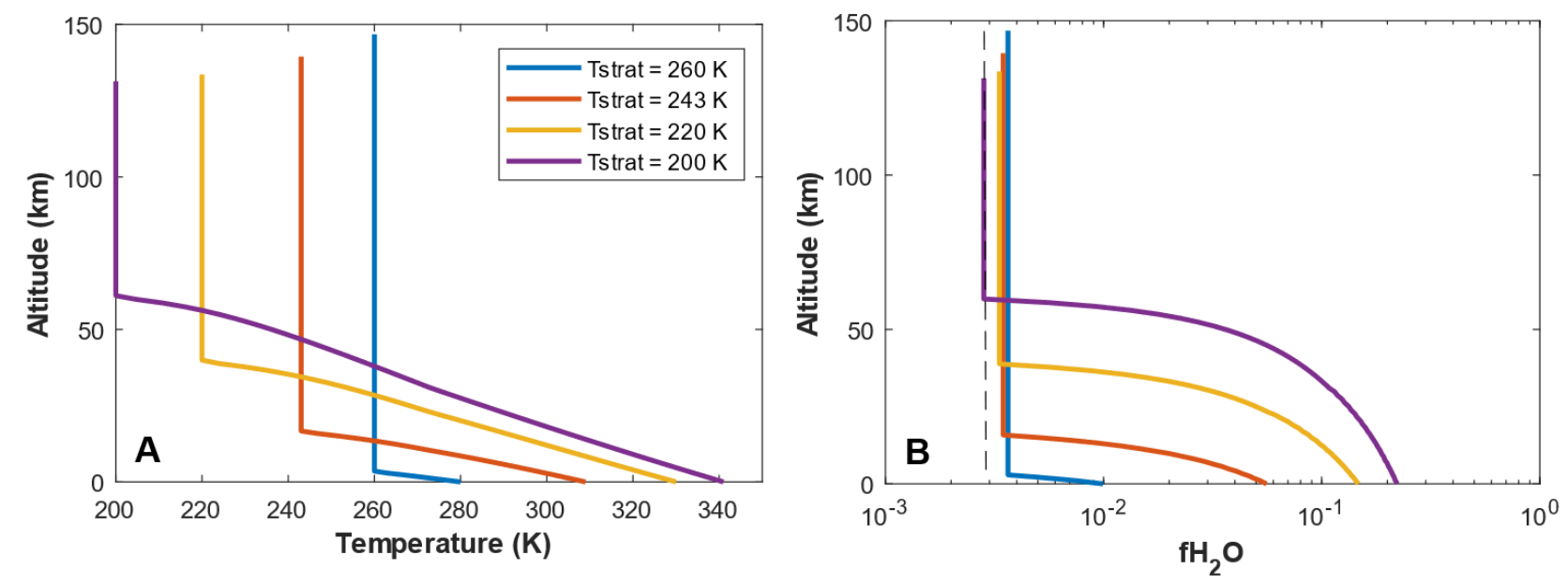

Figure 1: Vertical profiles of a) temperature and b) water vapor mixing ratio $\left(\mathrm{fH}_{2} \mathrm{O}\right)$ for different stratospheric temperature assumptions $(200,220,243$ and $260 \mathrm{~K})$. A moist greenhouse is triggered at stratospheric $\mathrm{fH}_{2} \mathrm{O}>\sim 3 \times 10^{-3}$ (dashed). 


\section{DISCUSSION}

Thus, at higher $\mathrm{T}_{\text {strat }}$, the atmosphere holds more water vapor at higher altitudes above the cold trap (consistent with the Clausius-Clapeyron equation), which accelerates the transition to a moist greenhouse. In 3-D models, this is represented by enhanced upward transport of water vapor.

Thus, most discrepancies with the moist greenhouse computation are likely not due to perceived deficiencies in 1-D (or 3-D) models, but it helps to understand why certain assumptions in 1-D models were made. The $T_{\text {strat }}=200 \mathrm{~K}$ assumption is convenient for $\mathrm{HZ}$ calculations because we do not know whether habitable $\mathrm{M}$-star planets with relatively low $\mathrm{T}_{\text {strat }}$ do not exist, perhaps under different atmospheric and planetary assumptions.

Nevertheless, as $\mathrm{T}_{\text {strat }}$ is directly correlated to NIR absorption, which is generally more intense on $\mathrm{M}$-star planets, it would be possible to compute $\mathrm{T}_{\text {strat }}$ as a function of star type using basic physical principles, and in turn, determine new moist greenhouse thresholds for such planets. I should note that 1-D and 3-D models are often consistent with one another when an appropriate methodology is employed.

Unfortunately, on those M-star planets in which $\mathrm{T}_{\text {strat }}$ is high, their habitability is in doubt, however. If such planets are currently undergoing moist greenhouses they would likely be desiccated. Unless originally located far away, these worlds would have lost tens - hundreds of Earth oceans during the superluminous pre-main-sequence stellar phase, which can last over 2 Gyr (e.g. Ramirez and Kaltenegger, 2014; Luger and Barnes, 2015). Also, because M-star luminosity varies little during the main-sequence, these planets may have been in this moist greenhouse state for many Gyr. Thus, unless their water inventories (by mass) exceed a few tens $\%$ of their planetary mass (e.g. Levi et al. 2017), they may be dry. Assuming such desert worlds are habitable, spectral features may be too weak to be detected.

\section{CONCLUSION}

Both 1-D and 3-D climate modelers should collaborate to understand both the common points and differences in our approaches. We can learn a lot from one another on the moist greenhouse problem among others.

ACKNOWLEDGEMENTS: I acknowledge enlightening discussions regarding the implications of GCM results with Eric Wolf, Yuka Fujii, and Tony Del Genio. I also acknowledge support from the Earth-Life Science Institute. 


\section{REFERENCES}

Fujii, Y., Del Genio, A.D., \& Amundsen, D.S. 2017, ApJ, 848, 2

Kasting, J.F., Whitmire, D.P, \& Reynolds, R.T. 1993, Icar, 101,1.

Kopparapu et al. 2017, ApJ, 845, 1.

Levi, A., Sasselov, D., \& Podolak, M. 2017, ApJ, 838(1) ,24

Luger, R., Barnes, R. 2015, AsBio 15.2

Ramirez, R. M., \& Kaltenegger, L. 2014, ApJL, 797, L25

Ramirez, R. M., \& Kaltenegger, L. 2017, ApJL, 837(1), L4 